\title{
Clinical utility of the Taylor spatial frame for limb deformities
}

\section{Doron Keshet \\ Mark Eidelman}

Pediatric Orthopedics Unit, Rambam Health Care Center, Haifa, Israel
Correspondence: Doron Keshet Pediatric Orthopedics Unit, Rambam Health Care Center, P O Box 9602, Haifa, Israel

Tel +972502061944

Email doron.keshet@gmail.com
This article was published in the following Dove Press journal:

Orthopedic Research and Reviews

30 May 2017

Number of times this article has been viewed
Abstract: The Taylor spatial frame (TSF) is a hexapod external fixator that can correct sixaxis deformities. The mathematical base of all hexapod systems is projective geometry, which describes complex repositioning of an object in space. The Taylor brothers developed one of the first six-axis correction systems, which is known today as TSF. Over the years, this system has become the most used six-axis deformity correction device. In this review, we describe the history behind TSF development, and describe the principles and clinical utility for application of the TSF in different settings, such as acute trauma, malunions, and various deformities of the lower and upper limb.

Keywords: external fixator, deformity correction, hexapod

\section{Introduction}

The Taylor spatial frame (TSF) is a modern hexapod external fixator that is able to correct six-axis deformities simultaneously using a virtual hinge. This frame was designed and created by the Taylor brothers in 1994 and has become one of the most popular external fixation systems.

\section{History of the TSF}

The history behind the TSF is fascinating and could easily be adopted as a movie script. Projective geometry is the mathematical basis of the hexapod system that is required for complex repositioning of an object in space. ${ }^{1}$ Chasles and Poinsot rediscovered and developed projective geometry to what we know today, but Gerard Desargues (Figure 1), a self-educated engineer, was the first to publish a manuscript on projective geometry in 1639. ${ }^{1,2}$ A year later, in 1640, a well-known French mathematician Blaise Pascal published his theorem on projective geometry, but this work was lost. Fortunately, Pascal's student, Philipe de la Hire made a copy of Pascal's book that was discovered by Michel Chasles in a bookshop after more than a century! $!^{2}$ Stewart designed a mechanism with six degrees of freedom known as Stewart platform that was later used to simulate flight conditions. The Stewart platform with two rings and six legs (hexapods) is extensively used in many fields of industry (robotics, flight simulators, etc.). An orthopedic surgeon from Elvis Presley Memorial Hospital, in Memphis, Tennessee, Charles Taylor and his brother Harold, an engineer, developed in 1994 a computer program and an external fixator that is known today as the TSF. The first operation using a TSF device was performed in 1995, and the device was patented in 1997. From 2002, a web-based program became available, with several 


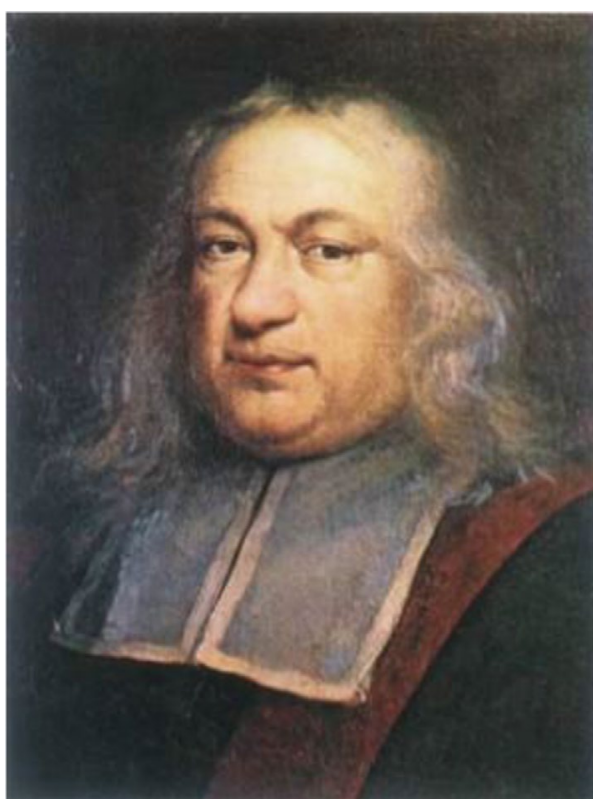

Figure I Gerard Desargues.

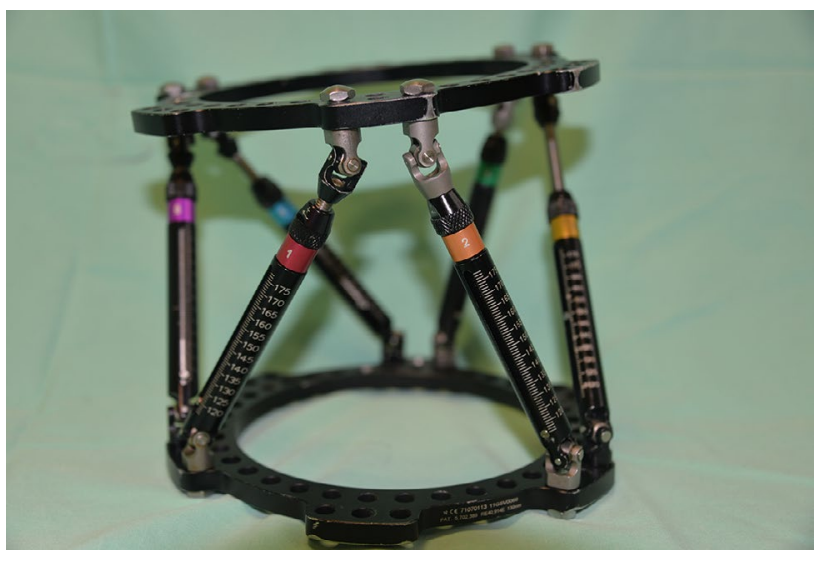

Figure 2 Standard TSF construction.

Abbreviation: TSF, Taylor spatial frame.

improvements and modifications done over the years. Many other hexapod systems entered the global market, but the TSF is undoubtedly the most used six-axis deformity device.

\section{Principles}

In order to apply a TSF on the limb, the surgeon must know several basic principles of the TSF system and must be able to perform deformity analysis. ${ }^{2,3}$

The basic TSF construction consists of two full or partial rings connected by six telescopic struts attached at special universal joints (Figure 2). By adjusting strut lengths, one ring can be repositioned with respect to the other. TSF pre-planning includes calculation of three groups of parameters, deformity, mounting and frame parameters. Deformity parameters show the relation between origin and corresponding point (Figure 3). The origin is always located on the reference fragment (either proximal or distal). Corresponding points, therefore, are always located on the moving fragment. In most cases, deformity parameters can be calculated on the basis of the preoperative X-rays. Deformity parameters include anteroposterior and lateral views of angulation and translation, axial view angulation and axial translation (which determines shortening or lengthening of the given case). Rotational deformity is determined by clinical examination. Mounting parameters reflect the relationship between the reference ring (the ring applied to the reference fragment) and the origin. Frame parameters define information on the diameters of the rings and lengths of the struts. Finally, the surgeon chooses the structure at risk (the structure that will undergo the most risky elongation during deformity correction) and safe velocity correction (usually $\sim 1 \mathrm{~mm}$ per day).

\section{Trauma application}

Acute application of a TSF in trauma is most commonly used for tibial fractures, and it can be used to stabilize closed or open fractures. ${ }^{4-7}$ When treating trauma of the lower limbs, a circular external fixator has the advantages of minimal soft tissue disruption and early weight bearing when compared with standard means of fracture fixation. The TSF uses the same basic principles as the Ilizarov external fixator for the treatment of fractures in the acute and delayed setting and posttraumatic deformities.

There is no consensus among surgeons regarding the correct configuration of half pins and wires per fixation block (bone segment above and below the fracture or the osteotomy). As a general rule, a minimum of three half pins and one Ilizarov wire are needed to achieve a stable fixation (Figure 4). With fracture healing, it is advised to gradually remove half pins and wires to create dynamization and reduce the risk of re-fractures.

The hexapod frame allows anatomic realignment of fractures; therefore, malunions are usually related to inaccurate input of deformity and/or mounting parameters. Before accomplishing bony union at the fracture site, it is possible to "run" the residual deformity program to achieve "finetuning" of bone alignment.

The TSF may be applied in two ways for fracture reduction. ${ }^{7}$ In the "rings first" method, each ring is mounted independently in an orthogonal manner to its respective fracture fragment. Once attached, the two rings can be used to manually reduce the fracture. The struts are then inserted between the two rings to hold the reduction; residual malalignment can be later corrected using the total residual program. Another 


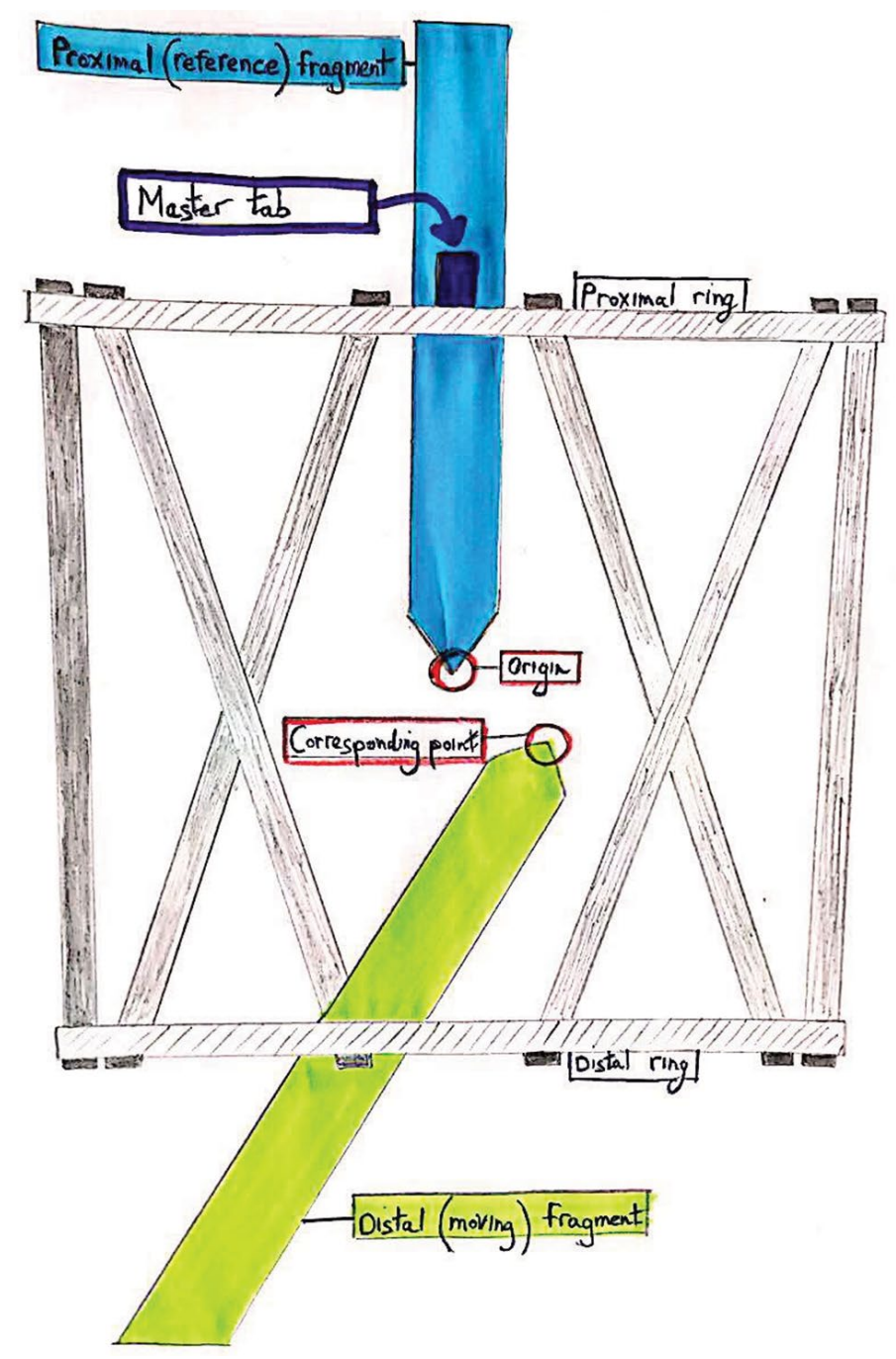

Figure 3 Deformity parameters show the relation between origin and corresponding points.

way to apply the TSF is the "modified first ring" method. After orthogonal applications of the rings, struts are left in an unlocked position. Distraction of the rings is done until they become parallel, and after fluoroscopic verification of fracture alignment, struts are locked. One can always perform a "fine-tuning" program during follow-up in the clinic.

Rozbruch et $\mathrm{al}^{8}$ described in 2008 the repair of tibial nonunions and bone defects using the TSF. Using intentional posterior angulation and shortening, it is possible to treat relatively large bone and soft tissue defects. Any residual shortening of the affected bone can be treated by an additional osteotomy above or below the defect and lengthening to compensate for shortening (Figure 5A-E).

\section{Double-level deformities using the TSF}

Common indications for double-level deformity correction are posttraumatic malunions, congenital limb deformities, chronic osteomyelitis sequelae and growth arrest of the physis in immature patients. Often, the deformity is corrected at one level, and the second level is used to correct only length (Figure 6A-E).

Double-level deformity correction can be performed in any limb but is most often done in the tibia and the femur. The typical ring configuration for double-level correction is a three-ring frame applied in such a manner that the middle ring is the reference for the proximal and distal segments of the bone.

Double-level correction can be performed in different ways:

A. Simultaneous double-level correction.

B. Staged correction: correction of one level first, then correction of the second level.

C. Acute correction of one level, then gradual correction of the second level. 


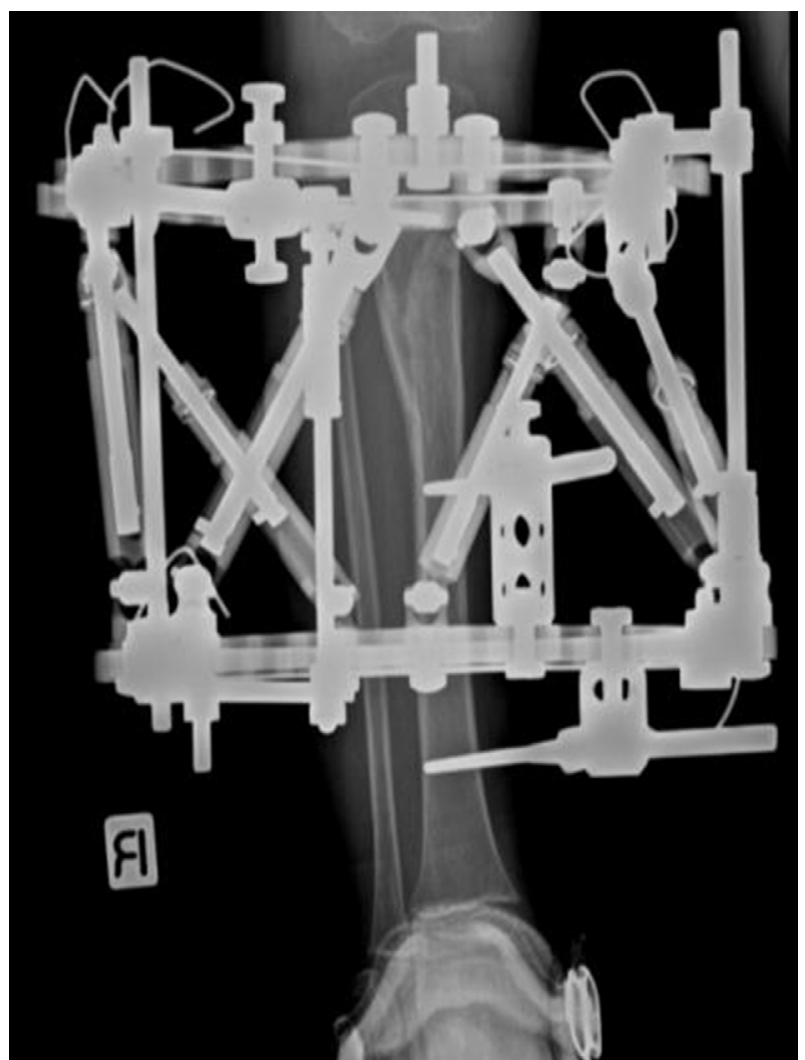

Figure 4 Tibial fracture treated with TSF.

Note: Note intensional varus angulation in order to prevent Cozen phenomenon (proximal tibial valgus deformation).

Abbreviation: TSF, Taylor spatial frame.

Before application of the TSF, precise deformity analysis must be performed to find both center of rotation of angulation (CORA) and the level of the osteotomies and their relations to CORA. The surgeon must also take under consideration proper rings and struts size, and level of the fibular osteotomy if needed, to estimate frame stability in order to decide if foot fixation is required.

\section{Blount disease}

Adolescent tibia vara (Blount's disease) is one of the most common reasons for TSF application, as TSF is a real "Blount's machine". Patients usually present during their second decade of life with marked genu varum, procurvatum deformity of the proximal tibia and internal tibial torsion. This combination of deformities in coronal, sagittal and axial planes results in a complex three-dimensional deformity making the TSF an ideal surgical treatment option.

Feldman et al treated 22 tibias with infantile and adolescent Blount with gradual correction using the TSF and in 21 achieved the desired correction goal with good clinical results and minimal complications. The authors concluded that six-axis deformity analysis and TSF provide accurate and safe correction of infantile and adolescent tibia vara. ${ }^{9}$

Standard treatment of adolescent tibia vara is a proximal tibial osteotomy and fibular osteotomy. In patients with proximal tibial varus, the correction can be carried out without fibular osteotomy if the patient does not have significant rotation or significant procurvatum. ${ }^{10}$

The need for fibular osteotomy in conjunction with proximal tibia osteotomy was discussed in several studies. In 2008, Eidelman et al published a series of eight patients with adolescent Blount that were treated with tibia osteotomy only and gradual correction with TSF and showed accurate deformity correction using the proximal tibiofibular joint as

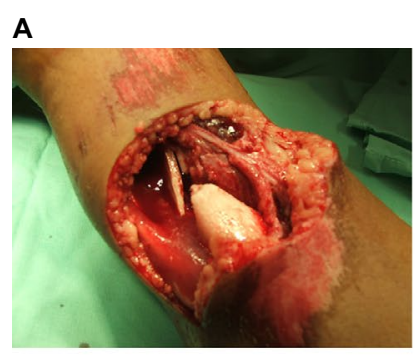

D
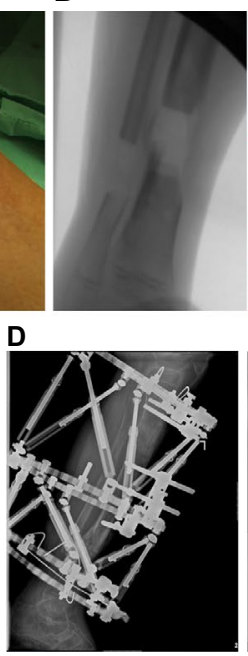

C
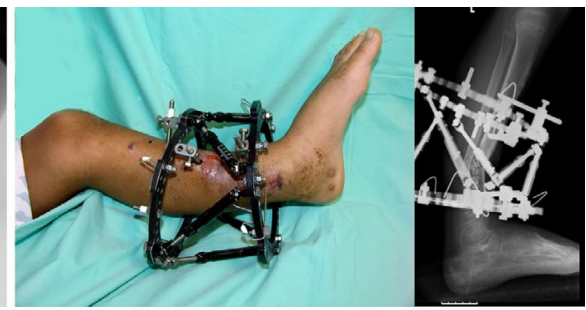

E

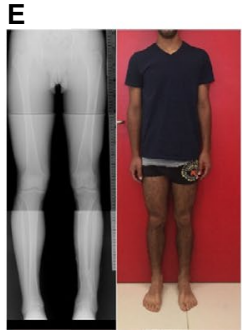

Figure 5 (A) Severe bone and tissue loss of a midtibial fracture in a 10-year-old boy. (B) A 14 mm bone defect after debridement. (C) Intensional posterior angulation for creation of bone shortening and wound closure. (D) Application of a third ring proximally with proximal bone transport and correction of alignment. (E) Clinical and radiographic appearance after 10 years of follow-up. 

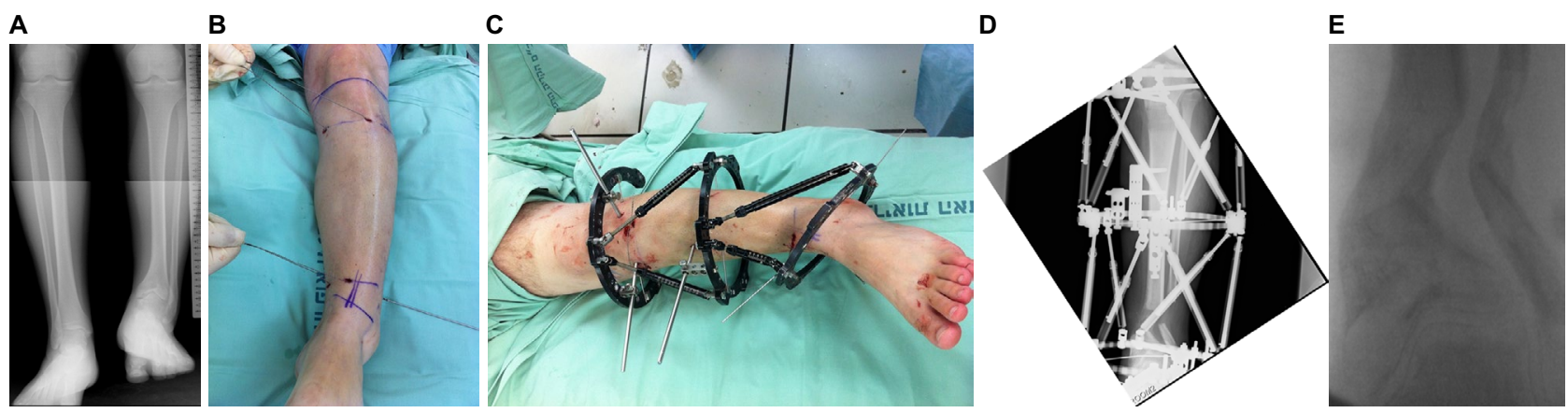

Figure 6 (A) Posttraumatic malunion of the distal tibia with $6 \mathrm{~cm}$ of shortening. (B) Double-level Gigli saw osteotomy. (C) Application of a three-ring TSF construction. (D) After simultaneous proximal lengthening and distal deformity correction. (E) The distal tibia after deformity correction.

Abbreviation: TSF, Taylor spatial frame.

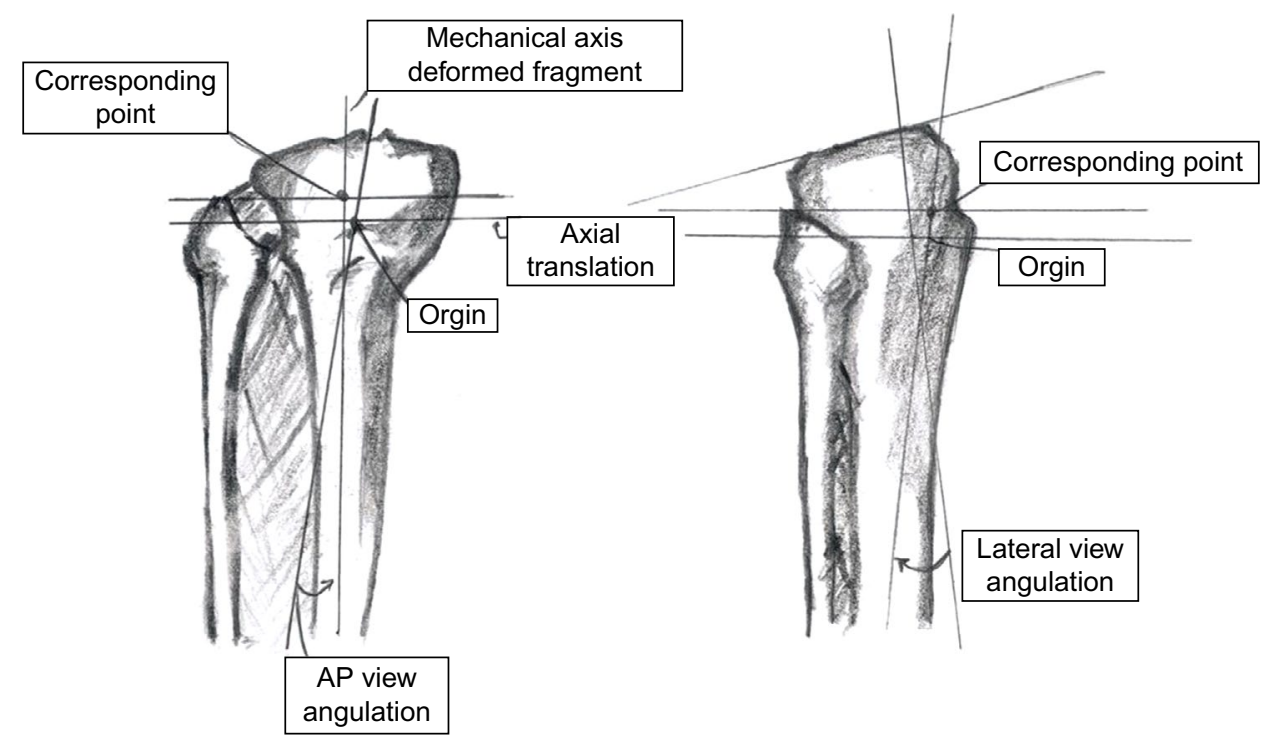

Figure 7 The origin is placed along the mechanical axis of reference fragment at the level of the proximal tibial-fibular joint.

Abbreviation: AP, anteroposterior.

the origin when planning the deformity correction (Figure 7). Sachs et al compared the results of 11 patients treated for adolescent Blount with tibia and fibular osteotomy and 15 patients treated with tibia osteotomy only. Their reports showed similar good results, and they concluded that in patients with adolescent tibia vara, correction may be performed safely without osteotomy and fixation of the fibula. ${ }^{11,12}$

Typical application of the TSF in adolescent Blounts includes passage of a Gigli saw around the tibia just distal to the proximal tibial tubercle (Figure 8). The proximal portion of the tibia is fixated to the proximal ring using a single $1.8 \mathrm{~mm}$ Ilizarov wire and three $6 \mathrm{~mm}$ half pins. The distal part of the tibia is then fixated to the distal ring with three or four $6 \mathrm{~mm}$ half pins and $1.8 \mathrm{~mm}$ wire (Figure 9). The osteotomy is completed using the Gigli saw. Typically, internal rotation is $<20^{\circ}$; therefore, there is no need to per- form fibular osteotomy. The correction is started 1 week after application of the TSF with correction velocity set at $0.75 \mathrm{~mm}$ daily (Figure 10).

Treatment of neglected Juvenile Blount's disease might be a challenging surgical problem that necessitates complete proximal tibia and fibula epiphysiodesis, elevation of the medial tibial plateau, proximal tibial osteotomy, preemptive tibial lengthening and overcorrection to valgus. Regardless of the treatment method chosen, recurrence of varus is common and further correction might be required close to maturity (Figure 11).

\section{Femoral application}

The use of TSF in the femur is more challenging as a result of patient inconvenience, due to a frame on the thigh and limitation of hip and knee motion. For decreasing patient 


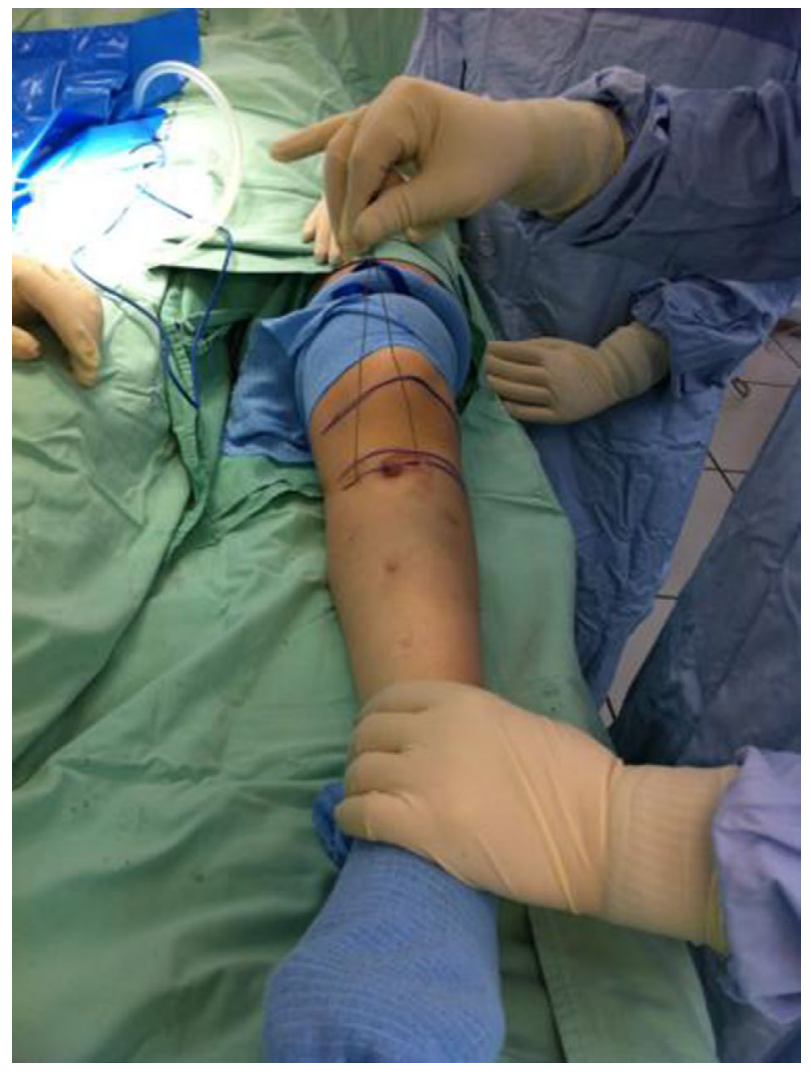

Figure 8 Gigli saw passed below tibial tubercle before frame application.

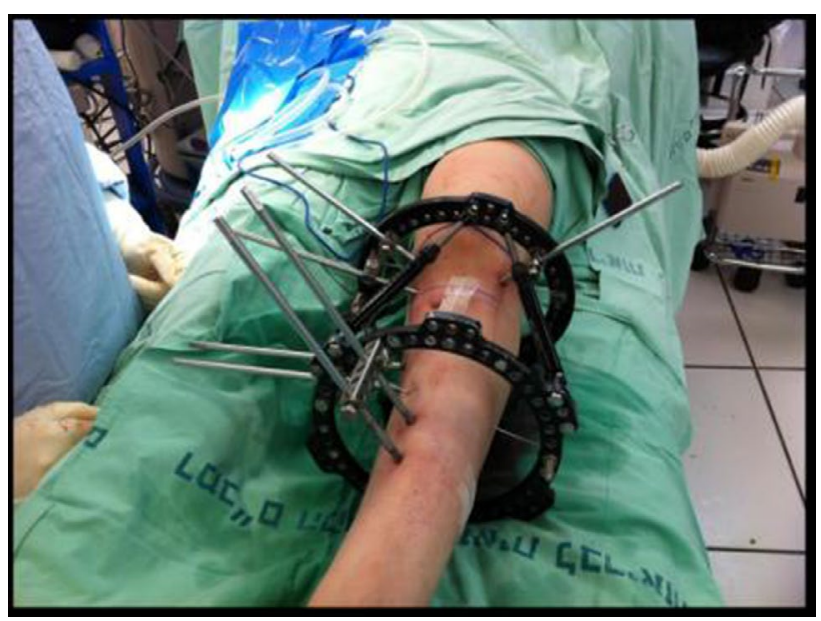

Figure 9 After TSF application.

Abbreviation: TSF, Taylor spatial frame.

discomfort, one can use a $2 / 3$ open ring on the posterior aspect for the distal ring to allow knee flexion and a $2 / 3$ open ring medially for the proximal ring to allow hip adduction. In such configuration, the surgeon must remember that the antimaster tab is situated $60^{\circ}$ externally (in cases where a distal reference was chosen). Another challenge when applying TSF on the femur is creation of a stable distal block. Usually, the

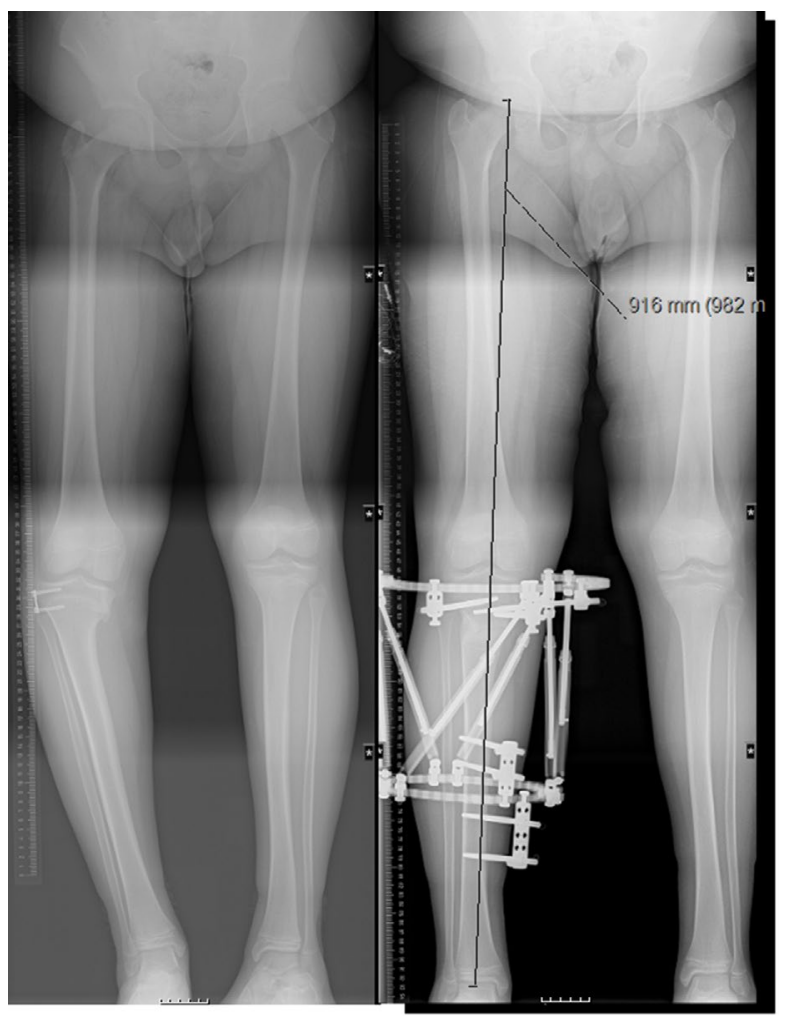

Figure 10 Radiographs before and after correction of adolescent Blount.

apex of the deformity is close to the knee joint, requiring a distal osteotomy. This creates a "geographical" and anatomic challenge for half pin insertion sometimes requiring use of wires that are less tolerated by patients.

There are a variety of etiologies described in the orthopedic literature as underlying causes of femoral deformity: congenital femur deficiency, fibrous dysplasia, multiple enchondromatosis, rickets, spondyloepiphyseal dysplasia, idiopathic deformity and osteomyelitis ${ }^{13}$ (Figure 12).

\section{Foot application}

The TSF can be used in a variety of cases of congenital and acquired deformities of the foot and ankle. Use of the TSF for correction of foot deformities has become popular with various frame configurations and foot programs that were developed recently. Currently, surgeons can choose one of the available programs to correct foot and ankle deformities (6×6 Miter; 6×6 Butt; ankle mode, etc.). Several frame options are available for treating foot and ankle deformities:

a. Standard frame with two regular rings connected by six struts (usually used for the correction of equinus and clubfoot-like deformities).

b. Miter frame.

c. Butt frame. 

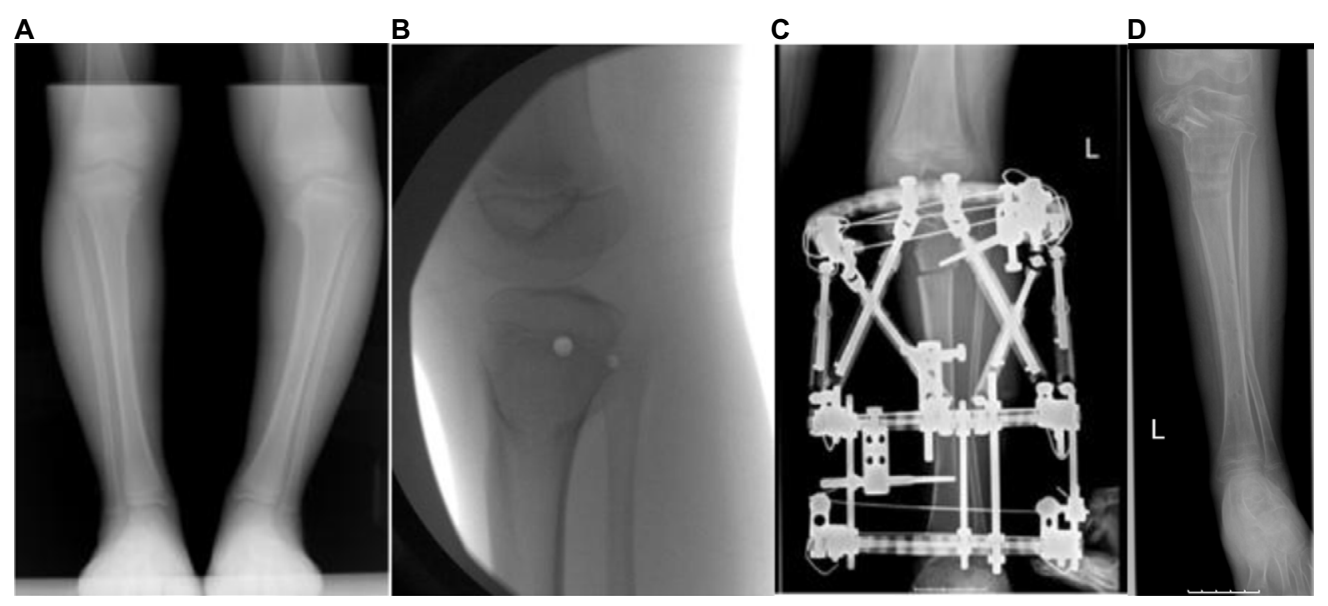

Figure I I (A) Juvenile tibia vara in a 5-year-old girl with medial bone bridge. (B) Proximal tibia + fibula epiphysiodesis in order to prevent deformity recurrence. (C) Tibial plateau elevation and proximal tibial osteotomy. (D) Final radiographic appearance after preemptive lengthening and overcorrection of tibia to valgus.

A

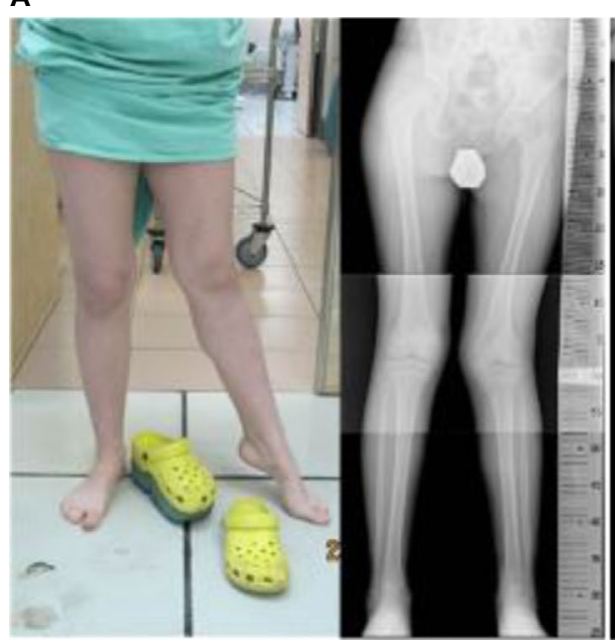

B

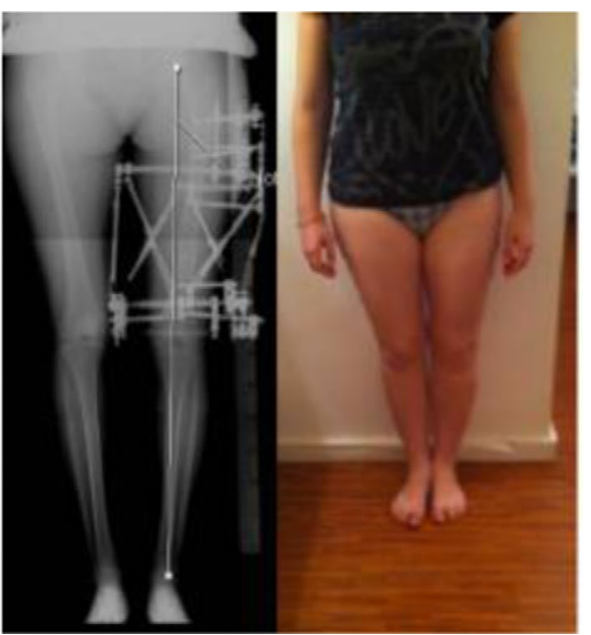

Figure 12 (A) Partial growth arrest of the distal femur after septic arthritis with $10 \mathrm{~cm}$ projected leg length discrepancy. (B) After $10 \mathrm{~cm}$ lengthening and deformity correction.

The standard frame is very useful and an "easy-to-use" construction. By using "ankle mode", one can correct equinus deformities using soft tissue distractions (Figure 13). In many cases of rigid foot deformities, usually patients with arthrogryposis or neglected clubfoot, a plantigrade foot can be achieved only after supramalleolar osteotomy and posterior angulation technique. ${ }^{14}$ The same standard frame can be applied in cases of neglected clubfoot deformities using the "PonseTaylor method". This method follows the same principles as the Ponseti protocol for serial casting using the TSF and has two stages of correction. The first stage of correction consists of adduction and hindfoot varus correction by rotation of the foot around the talus (olive wire inserted to the talar neck and attached to the proximal ring). At this stage, foot equinus is corrected to neutral dorsiflexion only. At the second stage, the foot is dorsiflexed to correct equinus (talar wire reattached to the distal ring) (Figure 14).

The Miter frame consists of two full rings, one $2 / 3$ ring and 12 struts (Figure 15). This frame allows correction of hindfoot and forefoot deformities as well as supramalleolar deformities as needed.

The Butt frame consists of a U-plate over the foot connected to standard rings over the tibia and foot. This configuration allows correction of midfoot and forefoot deformities alone or in combination with correction at a more proximal level (supramalleolar level). Usually, corrections initiated after midfoot osteotomies (cuboid and cuneiforms) are carried out by percutaneous Gigli saw. Technically, the most challenging part of the procedure is fixation of the distal ring to the forefoot. Two wires are inserted through the metatar- 


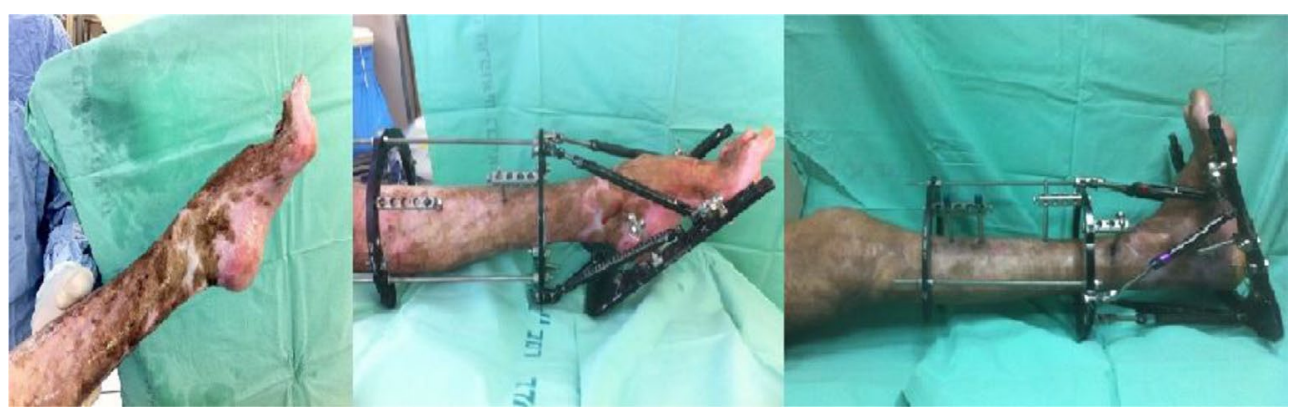

Figure 13 Post burn equinus treated with TSF with soft tissue distraction. Abbreviation: TSF, Taylor spatial frame.

A

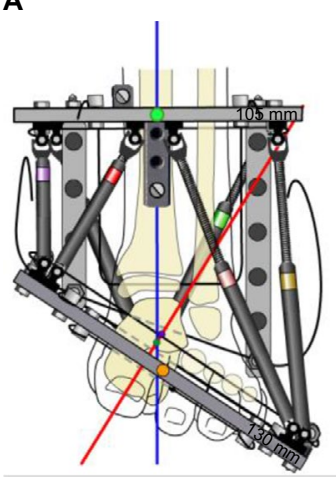

B

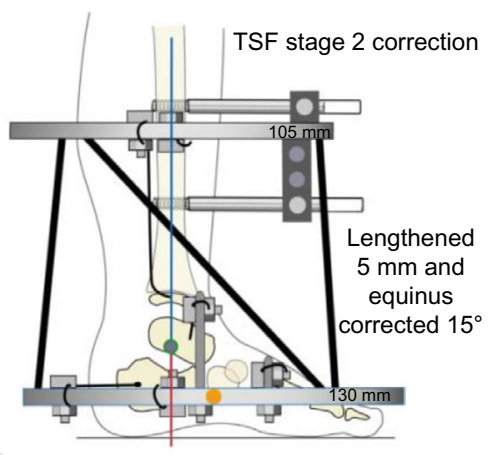

Figure 14 The "PonseTaylor" method. (A) After application of TSF. (B) After the first correction stage.

Note: Used with permission, Copyright 2017, Rubin institute for advanced orthopedics, Sinai hospital of Baltimore.

Abbreviation: TSF, Taylor spatial frame.

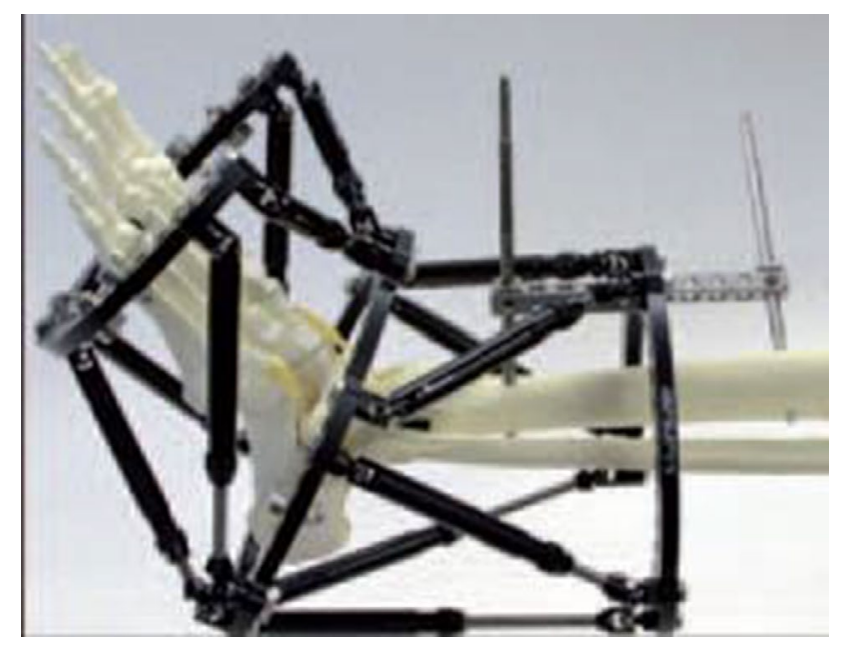

Figure 15 Miter frame.

sals: one through the first and second metatarsals and another through the third, fourth and fifth metatarsals. It is advisable to fixate the distal ring as distally as possible, using long posts attached to the wire, because there is no room available while fixating the distal ring at the level of the metatarsals. Only after fixation of the ring and applying all the struts can one insert the second wire to prevent "impingement" of this wire with the struts. After completion of fixation and removal of only two struts, the Gigli saw osteotomy can be completed under protection of the periosteal elevators. Finally, two additional so-called "stirrup" wires are inserted without tension above and below the osteotomy to prevent inadvertent correction outside the osteotomy.

Patients with severe residual clubfoot can present with toe flexion deformities and/or metatarso-phalangeal instability. To prevent worsening of these deformities, we recommend prophylactic pinning of the toes using thin Kirshner wires attached to the distal ring (Figure 16).

\section{TSF in the upper extremity}

The use of TSF in the upper extremities is less common, and there are only few articles that describe application of TSF on the arm and forearm. ${ }^{15-17}$ Fixation of the upper extremity might be a challenging task even for the experienced surgeon due to the anatomical complexity of the upper limb. In the humerus, the proximity of the neuromuscular leaves only limited area for pin insertion, and together with the presence of two bones with narrow diameters in the forearm, it creates a challenge for stable fixation.

One of the most common indications for TSF use is correction of cubitus varus, which is a three-dimensional deformity with varus, internal rotation and extension. While acute correction is a common procedure practice, there are common described complications: under and over correction, nonunion, prominence of the lateral condyle, growth arrest, osteomyelitis and neurological damage. Acute correction is also usually involved with relatively large exposure and challenging fixation. The main advantage of TSF is that all dimensions of the deformity are treated simultaneously, and in case of under correction, one can run residual program. Recently, the use of TSF was described for the correction of 


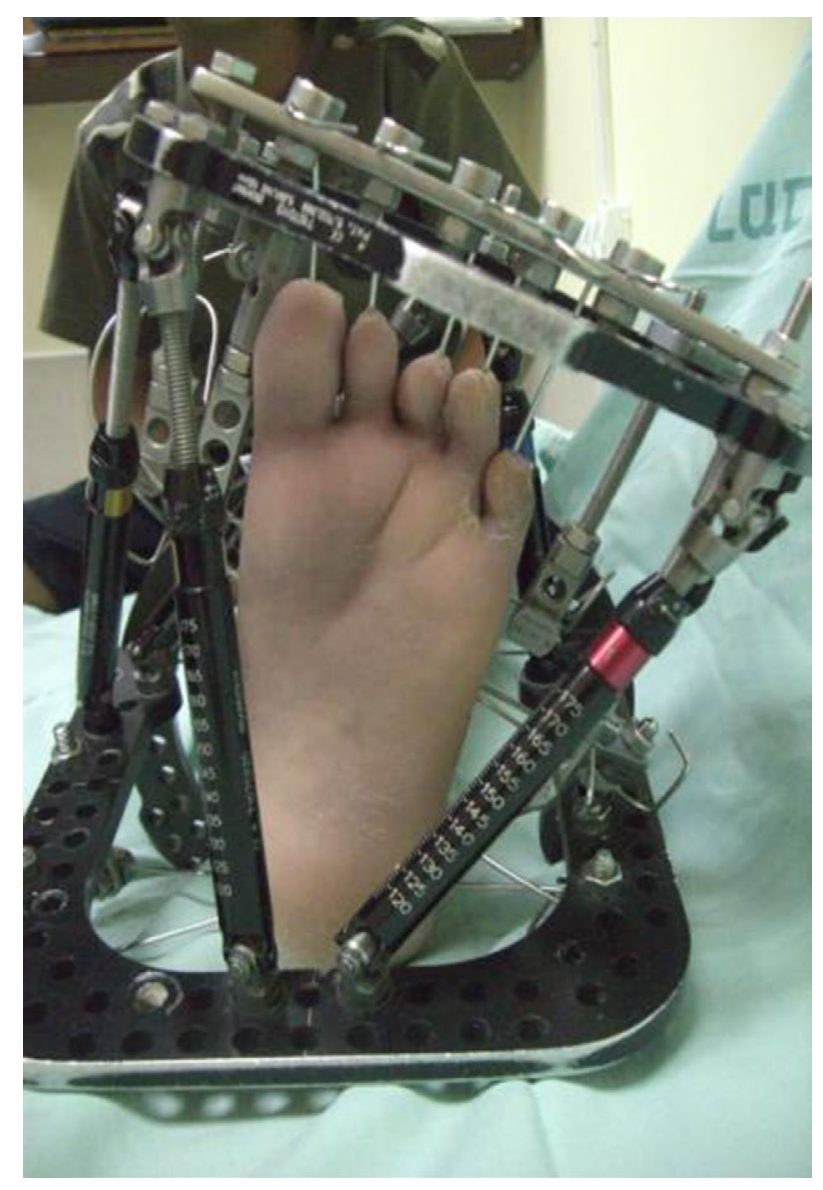

Figure 16 Prophylactic pinning of the toes using thin Kirshner wires attached to the distal ring (Butt frame construction).

cubitus varus in children and adults. ${ }^{16}$ The article explained in details a new method of fixation of the distal humerus (Herzenberg method) that allows a very distal humeral osteotomy, close to the apex of the deformity (Figure 17). Overall patient satisfaction after the procedure was high (Figure 18).

Several articles described use of TSF in distal radius and ulna malunions. ${ }^{15,17}$ Al-Sayyad ${ }^{17}$ described his experience in treating children with TSF in the upper extremity and concluded that TSF can be a good surgical solution as definitive treatment for upper extremity conditions involving deformity, shortening or bone transport in the pediatric and adolescent population.

\section{Discussion}

The Ilizarov method of deformity correction and limb lengthening was the most important contribution in the field of deformity correction in the last century. ${ }^{2,18,19}$ This method remains the basis for deformity correction using internal and external fixation. Correction of deformities can be done using monolateral or circular fixators. While monolateral fixators are

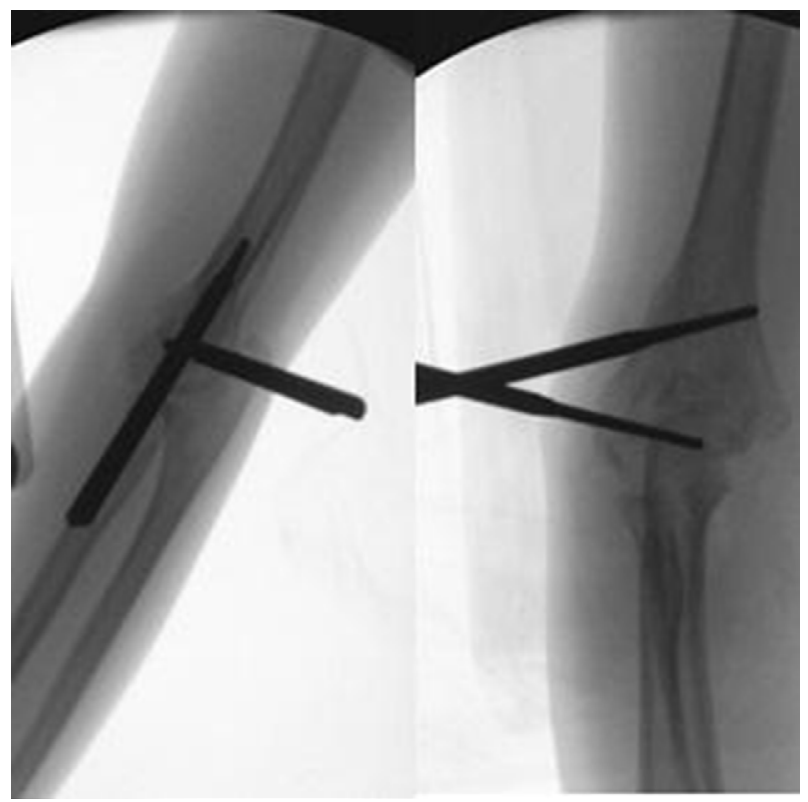

Figure 17 Pins inserted in delta configuration in sagittal and coronal planes.
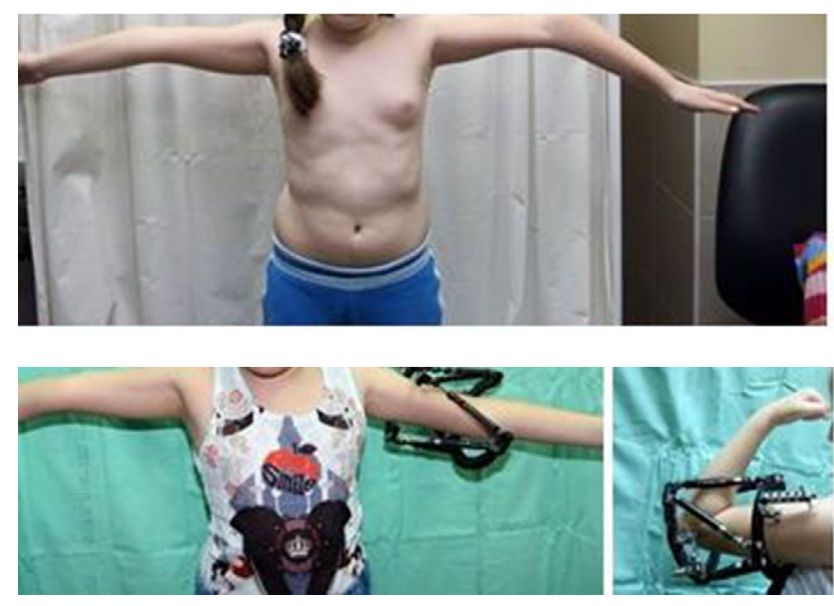

Figure 18 Severe left cubitus varus after malunion of supracondylar fracture of the distal humerus treated with TSF.

Abbreviation: TSF, Taylor spatial frame.

more comfortable and less bulky, in many cases, they are not suitable for successful and stable correction. Significant disadvantages of the Ilizarov frame include a long learning curve and the need for frame adjustments and creation of additional hinges when correcting multiplanar deformities. Furthermore, correction of rotational deformities with the Ilizarov frame is a challenging task even for the most experienced surgeons. ${ }^{15,20,21}$ The greatest advantage of the TSF and other hexapod systems is the elimination of the need for frame adjustments because the simplest and most complex deformities are treated using the same frame. TSF is able to correct six-axis deformities 
simultaneously with computer accuracy. Therefore, after application of the frame all that is left for the surgeon to do is to perform accurate deformity analysis and insert correct data to the Web site program. Undoubtedly, hexapod systems have become the treatment of choice for multiplanar skeletal deformities, especially with rotational components.

We carefully reviewed the literature over the last 15 years and found that most publications described deformity corrections using TSF around the tibia. To a lesser extent, we found publications describing femoral, foot and upper extremity application. There is general consensus that the TSF is a very efficient surgical tool that allows correction of any kind of deformity with computer accuracy. ${ }^{8,15,22,23}$

Manner et $\mathrm{al}^{23}$ compared the results of complex deformity correction in 155 patients using TSF and Ilizarov frames and found that the goal of correction was achieved in $91 \%$ of patients treated with a TSF compared to $56 \%$ of patients treated with an Ilizarov fixator.

In order to become a master of the Ilizarov method using the Ilizarov frame, one must go through years of training, and even so, when dealing with multiplanar deformities, it can be a challenge for the most experienced surgeon. In contrast to becoming a hexapod master can happen rather fast, a fact that can easily explain the worldwide rising popularity of TSF and other hexapod systems.

The development of internal lengthening nails and especially magnetic nails has dramatically changed our approach to limb lengthening and deformity correction. ${ }^{24-26}$ Internal lengthening changed the rules of the game and is a true revolution in the field of limb lengthening and deformity correction. Internal lengthening significantly reduces the lengthening process and reduces pain and all problems related to external fixation (pin tract infection, etc.). Although internal lengthening is a powerful surgical option for correction of various deformities, the use of this method in immature patients is limited due to the proximity of growth plates. The hexapod external fixator therefore remains an important surgical tool and the working horse for lengthening and deformity correction of the lower limbs, especially in children and adolescents.

\section{Acknowledgments}

Neither this manuscript nor substantial parts of it are under consideration for publication elsewhere, been published or made available elsewhere in a manner that could be construed as a prior or duplicate publication of the same content. There is neither a manuscript of related content under consideration for publication elsewhere nor has one been published or made available elsewhere. Neither I nor the co-author have patents, whether planned, pending or issued, broadly relevant to the work. Written informed consent was obtained from all patients and parents whose photos were used in this review.

\section{Disclosure}

The authors report no conflicts of interest in this work.

\section{References}

1. Paley D. History and science behind the six-axis correction external fixation devices in Orthopedic Surgery. Oper Tech Orthop. 2011;21: 125-128.

2. Paley D, editor. Principles of Deformity Correction. Berlin, Heidelberg: Springer; 2002.

3. Standard S, Herzenberg J, Conway J, Siddiqui N. The Art of Limb Alignment. 2nd ed. Baltimore, Maryland : Rubin Institute for Advanced Orthopedics, Sinai Hospital of Baltimore; 2012.

4. Al-Sayaad MJ. Taylor spatial frame in the treatment of pediatric and adolescent tibial shaft fractures. J Pediatr Orthop. 2006;26(2): 164-170.

5. Zenios M. The use of the Taylor spatial frame for the treatment of unstable tibial fractures in children. J Orthop Trauma. 2013;27(10): 563-568.

6. Eidelman M, Katzman A. Treatment of complex tibial fractures in children with the Taylor spatial frame. Orthopedics. 2008;31(10):992-995.

7. Iobst SA. Hexapod external fixation for tibial fractures in children. J Pediatr Orthop. 2016;36(suppl 1):S24-S28.

8. Rozbruch SR, Pugsley JS, Fragomen AT, Ilizarov S. Repair of tibial nonunions and bone defects with the Taylor spatial frame. $J$ Orthop Trauma. 2008;22(2):88-95.

9. Feldman DS, Madan SS, Koval KJ, van Bosse HJ, Bazzi J, Lehman WB. Correction of tibia vara with six-axis deformity analysis and the Taylor spatial frame. J Pediatr Orthop. 2003;23(3):387-391.

10. Taylor JC [homepage on the Internet]. Correction of General Deformity with Taylor Spatial Frame. 2013. Available from: http://www.jcharlestaylor.com. Accessed December 2016.

11. Sachs O, Katzman A, Abu-Johar E, Eidelman M. Treatment of adolescent Blount disease using Taylor spatial frame with and without fibular osteotomy: is there any difference? J Pediatr Orthop. 2015;35(5): 501-506.

12. Eidelman M, Bialik V, Katzman A. The use of the Taylor spatial frame in adolescent Blount's disease: is fibular osteotomy necessary? J Child Orthop. 2008;2(3):199-204.

13. Marangoz S, Feldman DS, Sala DA, Hyman JE, Vitale MG. Femoral deformity correction in children and young adults using Taylor spatial frame. Clin Orthop Relat Res. 2008;466(12):3018-3024.

14. Eidelman M, Katzman A. Treatment of arthrogrypotic foot deformities with the Taylor spatial frame. J Pediatr Orthop. 2011;31(4): 429-434.

15. Eidelman M, Bialik V, Kazman A. Correction of deformities in children using the Taylor spatial frame. J Pediatr Orthop B. 2006;15(6): 387-395.

16. Belthur MV, Iobst CA, Bor N, et al. Correction of the cubitus varus after pediatric supracondylar fracture: alternative method using Taylor spatial frame. J Pediatr Orthop. 2016;36(6):608-617.

17. Al-Sayyad MJ. Taylor spatial frame in the treatment of upper extremity conditions. J Pediatr Orthop. 2012;32(2):169-178.

18. Ilizarov GA, editor. Transosseous Osteosynthesis: Theoretical and Clinical Regeneration of the Regeneration and Growth of Tissue. Berlin, Germany: Springer-Verlag; 1992.

19. Ilizarov GA. The principles of the Ilizarov method. Bull Hosp Jt Dis Orthop Inst. 1988;48(1):1-11.

20. Binski JC. Taylor spatial frame in acute fracture care. Tech Orthop. 2002;17:173-184. 
21. Birch JG, Samchukov ML. Use of the Ilizarov method to correct lower limb deformities in children and adolescents. J Am Acad Orthop Surg. 2004;12(3):144-154.

22. Koren L, Keren Y, Eidelman M. Multiplanar deformities correction using Taylor spatial frame in skeletally immature patients. Open Orthop J. 2016;10:71-79.

23. Manner HM, Huebl M, Radler C, Ganger R, Petje G, Grill F. Accuracy of complex lower-limb deformity correction with external fixation: a comparison of the Taylor spatial frame with the Ilizarov ring fixator. J Child Orthop. 2007;1(1):55-61.
24. Rozbruch SR, Birch JG, Dahl MT, Herzenberg JE. Motorized intramedullary nail for management of limb-length discrepancy and deformity. J Am Acad Orthop Surg. 2014;22(7):403-409.

25. Kirane YM, Fragomen AT, Rozbruch SR. Precision of PRECISE internal lengthening nail. Clin Orthop Relat Res. 2014;472(12): 3869-3878.

26. Black SR, Kwon MS, Cherkashin AM, Samchukov ML, Birch JG, Jo $\mathrm{CH}$. Lengthening in congenital femoral deficiency: a comparison of circular external fixation and a motorized intramedullary nail. $J$ Bone Joint Surg Am. 2015;97(17):1432-1440.
Orthopedic Research and Reviews

\section{Publish your work in this journal}

Orthopedic Research and Reviews is an international, peer-reviewed, open access journal that focusing on the patho-physiology of the musculoskeletal system, trauma, surgery and other corrective interventions to restore mobility and function. Advances in new technologies, materials, techniques and pharmacological agents are particularly

\section{Dovepress}

welcome. The manuscript management system is completely online and includes a very quick and fair peer-review system, which is all easy to use. Visit http://www.dovepress.com/testimonials.php to read real quotes from published authors. 